\title{
THE NAVIER-STOKES EQUATION WITH INHOMOGENEOUS BOUNDARY CONDITIONS BASED ON VORTICITY
}

\author{
JIŘÍ NEUSTUPA \\ Faculty of Mechanical Engineering, Czech Technical University \\ Karlovo nám. 13, 12135 Praha 2, Czech Republic \\ E-mail: neustupa@math.cas.cz \\ PATRICK PENEL \\ Departement de Mathématique et Laboratoire Systèmes Navals Complexes \\ Université du Sud-Toulon-Var \\ BP 20132, 83957 La Garde, France \\ E-mail: penel@univ-tln.fr
}

\begin{abstract}
We formulate a boundary value problem for the Navier-Stokes equations with prescribed $\boldsymbol{u} \cdot \boldsymbol{n}, \operatorname{curl} \boldsymbol{u} \cdot \boldsymbol{n}$ and alternatively $(\partial \boldsymbol{u} / \partial \boldsymbol{n}) \cdot \boldsymbol{n}$ or $\operatorname{curl}^{2} \boldsymbol{u} \cdot \boldsymbol{n}$ on the boundary. We deal with the question of existence of a steady weak solution.
\end{abstract}

1. Introduction and motivation. We deal with the steady Navier-Stokes system

$$
\begin{aligned}
-\nu \Delta \boldsymbol{u}+(\boldsymbol{u} \cdot \nabla) \boldsymbol{u}+\nabla p & =\boldsymbol{f}, \\
\operatorname{div} \boldsymbol{u} & =0,
\end{aligned}
$$

in a bounded domain $\Omega \in \mathbb{R}^{3}$. The system describes the motion of a viscous incompressible fluid with a constant density (we assume that it equals one). We denote by $\boldsymbol{u}$ the velocity, by $p$ the pressure, by $\boldsymbol{f}$ the specific body force and by $\nu$ the coefficient of viscosity. The equation (1) expresses the balance of momentum and the equation (2) represents the condition of incompressibility.

The system (1), (2) is usually considered with the homogeneous Dirichlet boundary condition

$$
\left.\boldsymbol{u}\right|_{\partial \Omega}=\mathbf{0}
$$

2000 Mathematics Subject Classification: Primary 35Q30; Secondary 76D05.

Key words and phrases: Navier-Stokes equations.

The paper is in final form and no version of it will be published elsewhere. 
in the case when $\partial \Omega$ is a fixed wall. This condition was suggested by G. G. Stokes in 1845 and it expresses the requirement that

$$
\text { (a) }\left.\boldsymbol{u} \cdot \boldsymbol{n}\right|_{\partial \Omega}=0, \quad \text { (b) }\left.\boldsymbol{u}_{\tau}\right|_{\partial \Omega}=\mathbf{0},
$$

where $\boldsymbol{u}_{\tau}$ denotes the projection of $\boldsymbol{u}$ onto the plane tangential to $\partial \Omega$. While the first condition (4a) naturally follows from the impermeability of the wall, the second part (4b) is often called the "no-slip-condition" because it is believed that the fluid cannot slip on the boundary due to its viscosity. It is known that the condition (3) is, for divergence-free vector fields $\boldsymbol{u}$ from $\boldsymbol{W}^{1,2}(\Omega)$, equivalent to three scalar conditions

$$
\text { (a) }\left.\boldsymbol{u} \cdot \boldsymbol{n}\right|_{\partial \Omega}=0
$$

(b) $\left.\operatorname{curl} \boldsymbol{u} \cdot \boldsymbol{n}\right|_{\partial \Omega}=0$,

(c) $\left.\frac{\partial \boldsymbol{u}}{\partial \boldsymbol{n}} \cdot \boldsymbol{n}\right|_{\partial \Omega}=0$,

see e.g. [11]. Note that the third condition (5c) means that the normal component of the viscous stress, acting on the boundary, is zero. It can be expressed by the equation $\boldsymbol{n} \cdot \mathbb{T}(\boldsymbol{u}) \cdot \boldsymbol{n}=0$, where $\mathbb{T}$ is the viscous stress tensor. For the incompressible isotropic Newtonian fluid, $\mathbb{T}$ satisfies $\mathbb{T}(\boldsymbol{u})=2 \nu(\nabla \boldsymbol{u})_{s}$ where $(\nabla \boldsymbol{u})_{s}$ denotes the symmetrized gradient of $\boldsymbol{u}$. Although some fundamental questions still remain open, it can be said that the theory of the system (1), (2) with the boundary condition (3) is relatively deeply elaborated.

H. Navier, coming from the molecular-kinetic theory of fluids, had already in 1824 proposed the boundary condition saying that the velocity on the boundary is proportional to the tangential component of the stress. This condition can be expressed by the equations

$$
\text { (a) }\left.\boldsymbol{u} \cdot \boldsymbol{n}\right|_{\partial \Omega}=0
$$

(b) $(\mathbb{T}(\boldsymbol{u}) \cdot \boldsymbol{n})_{\tau}+k \boldsymbol{u}=\mathbf{0}$,

where $(\mathbb{T}(\boldsymbol{u}) \cdot \boldsymbol{n})_{\tau}$ denotes the tangential component of the stress acting on the boundary and $k$ is the coefficient of proportionality. The condition $(6 \mathrm{~b})$ naturally follows from the weak formulation of the problem (1), (2) which reads: Given $\boldsymbol{f}$ in the dual $\boldsymbol{W}_{\sigma}^{-1,2}(\Omega)$ to the space $\boldsymbol{W}_{\sigma}^{1,2}(\Omega)$ (= the space of all divergence-free vector functions from $\boldsymbol{W}^{1,2}(\Omega)$ whose normal component on $\partial \Omega$ equals zero). We search for $\boldsymbol{u} \in \boldsymbol{W}_{\sigma}^{1,2}(\Omega)$ such that

$$
\int_{\Omega}[\mathbb{T}(\boldsymbol{u}) \cdot \nabla \boldsymbol{\phi}+(\boldsymbol{u} \cdot \nabla) \boldsymbol{u} \cdot \boldsymbol{\phi}] \mathrm{d} \boldsymbol{x}+\int_{\partial \Omega} k \boldsymbol{u} \cdot \boldsymbol{\phi} \mathrm{d} S=\langle\boldsymbol{f}, \boldsymbol{\phi}\rangle_{\Omega}
$$

for all $\boldsymbol{\phi} \in \boldsymbol{W}_{\sigma}^{1,2}(\Omega)$, where $\langle., .\rangle_{\Omega}$ is the duality between $\boldsymbol{W}_{\sigma}^{-1,2}(\Omega)$ and $\boldsymbol{W}_{\sigma}^{1,2}(\Omega)$. Indeed, if $\boldsymbol{u}$ is a "smooth" solution of this problem then, considering at first the test functions $\phi$ with a compact support in $\Omega$, we find out that there exists an appropriate pressure $p$ such that $\boldsymbol{u}, p$ satisfy the equation (1) at least a.e. in $\Omega$. Then, considering all possible test functions $\phi \in \boldsymbol{W}_{\sigma}^{1,2}(\Omega)$, and integrating by parts in (7), we arrive at the identity

$$
\int_{\partial \Omega}[\mathbb{T}(\boldsymbol{u}) \cdot \boldsymbol{n}+k \boldsymbol{u}] \cdot \boldsymbol{\phi} \mathrm{d} S=0
$$

which implies (6b). The inequality $k>0$ guarantees that the quadratic form

$$
\mathcal{A}(\boldsymbol{u}, \boldsymbol{u}):=\int_{\Omega} \mathbb{T}(\boldsymbol{u}) \cdot \nabla \boldsymbol{u} \mathrm{d} \boldsymbol{x}+\int_{\partial \Omega} k|\boldsymbol{u}|^{2} \mathrm{~d} S
$$

is coercive, which plays an important role in the proof of existence of a weak solution to the problem just formulated above. Navier's boundary condition has been considered 
in many papers, let us e.g. mention W. Jäger and A. Mikelič [7] and W. Zajączkowski [15]. These boundary conditions enable the fluid to slip on the boundary, which is realistic especially if the boundary is smooth. Here we mean "smooth" not only in the mathematical sense, but so that the scale of its eventual microscopic roughness is comparable with the size of the fluid particles. Under the assumptions that the law of the conservation of momentum holds "up to the boundary" and the friction between the fluid and the wall is proportional to $-\boldsymbol{u}$ (with the coefficient of proportionality $k$ ), one can really arrive at (6b), but the natural physical requirement is that $k=\kappa t_{n}$ where $\kappa$ is the coefficient of friction between the fluid and the wall and $t_{n}$ is the normal component of the total stress acting onto the boundary. Note that the important contribution to $\mathfrak{t}_{n}$, which complicates the analysis as well as numerical solution of the model, is made by the pressure: it is due to the form $-p \mathbb{I}+2 \nu(\nabla \boldsymbol{u})_{s}$ of the total stress tensor. Here $p$ appears explicitly, but it can also be contained implicitly in the viscosity coefficient $\nu$ - more details can be found in a series of papers on incompressible viscous fluids with pressure dependent viscosity by J. Málek, R. Rajagopal et al. (see e.g. [3]). The difficulties generally connected with the influence of boundary conditions were admitted by C. Truesdell in 1952, see [14], who acknowledged that the lack of proper boundary conditions represents a big problem in future development of mathematical fluid mechanics.

The comparison of Dirichlet's boundary condition (3) with Navier's boundary conditions (6) shows that while in the first case $\boldsymbol{u}=\mathbf{0}$ on the boundary, which is a strong requirement, in the second case the only actual geometrical condition we impose is (6a). (Condition (6b) follows from physical considerations.) This situation motivated us to study other boundary conditions which also admit the slippery of the fluid on the boundary, which stand in some sense "between (3) and (6)" and which enable us to develop the theory of the Navier-Stokes equation, similarly as e.g. the boundary condition (3). We have explained in several papers (see [1], [10], [11]) that a relatively consistent theory of the Navier-Stokes equation can be created with the "generalized impermeability boundary conditions"

$$
\text { (a) }\left.\boldsymbol{u} \cdot \boldsymbol{n}\right|_{\partial \Omega}=0
$$

(b) $\left.\operatorname{curl} \boldsymbol{u} \cdot \boldsymbol{n}\right|_{\partial \Omega}=0$,

(c) $\left.\operatorname{curl}^{2} \boldsymbol{u} \cdot \boldsymbol{n}\right|_{\partial \Omega}=0$

on a fixed wall $\partial \Omega$. These conditions also enable the fluid to slip on the boundary. The second condition ( $8 \mathrm{~b})$ implies that the "2D flow" $\left.\boldsymbol{u}\right|_{\partial \Omega}$ has no vortices on $\partial \Omega$. The third condition (8c) means that Div $\mathbb{T} \cdot \boldsymbol{n}=0$ on $\partial \Omega$, which says that not the normal component of viscous stress acting on the boundary is zero, like in the case of $(5 \mathrm{c})$, but the normal component of the rate of production of the viscous stress on the boundary equals zero. Only the first two boundary conditions (8a), (8b), which coincide with (5a), (5b), are used in the definition of a weak solution to the problem (1), (2), (8), see [1] and [11]. However, it is shown in [1] and [11] that if the weak solution is "smooth" then it also automatically satisfies (8c) as a natural condition which results from the appropriate weak formulation of the problem. (In the present paper, we explain in Sections 3 and 4 that there in fact exists certain freedom in the weak formulation, consisting in the construction of functions $\boldsymbol{a} \in \boldsymbol{W}^{1,2}(\Omega)$ and $\boldsymbol{b} \in \boldsymbol{W}^{-1 / 2,2}(\partial \Omega)$ and in the choice of admissible test functions, so that the resulting natural condition need not necessarily be only $(8 \mathrm{c})$ - it can also be inhomogeneous and it can be of the type (9c) or (10c).) We have further shown in [1] 
that the boundary conditions (8) induce the same series of boundary conditions for the vorticity $\boldsymbol{\omega}:=\operatorname{curl} \boldsymbol{u}$ of the flow. It is a big advantage in comparison with (3) or (6) because the latter conditions for $\boldsymbol{u}$ do not lead to a well posed boundary-value problem for $\boldsymbol{\omega}$.

In this paper, we focus on the equations (1), (2) either with the inhomogeneous form of the boundary conditions (5):
(a) $\left.\boldsymbol{u} \cdot \boldsymbol{n}\right|_{\partial \Omega}=\alpha_{0}$,

(b) $\left.\operatorname{curl} \boldsymbol{u} \cdot \boldsymbol{n}\right|_{\partial \Omega}=\alpha_{1}$,

(c) $\left.\frac{\partial \boldsymbol{u}}{\partial \boldsymbol{n}} \cdot \boldsymbol{n}\right|_{\partial \Omega}=\alpha_{2}$,

or with the inhomogeneous form of the boundary conditions (8):
(a) $\left.\boldsymbol{u} \cdot \boldsymbol{n}\right|_{\partial \Omega}=\alpha_{0}$,
(b) $\left.\operatorname{curl} \boldsymbol{u} \cdot \boldsymbol{n}\right|_{\partial \Omega}=\alpha_{1}$
(c) $\left.\operatorname{curl}^{2} \boldsymbol{u} \cdot \boldsymbol{n}\right|_{\partial \Omega}=\alpha_{2}$.

2. Auxiliary results. We assume that $\Omega$ is a bounded simply connected domain with the boundary of the class $C^{2,1}$. The case of a general non-smooth domain will be treated in a forthcoming paper [12]. By the simple connectedness of $\Omega$ we mean that each closed curve in $\Omega$ can be homotopically contracted to a point without ever leaving $\Omega$. However, the boundary of $\Omega$ can generally have more components $\Gamma_{0}, \Gamma_{1}, \ldots, \Gamma_{N}$ so that

$$
\Omega=\operatorname{Int} \Gamma_{0} \cap \operatorname{Ext} \Gamma_{1} \cap \ldots \cap \operatorname{Ext} \Gamma_{N} .
$$

We list some notation and auxiliary results from [1] and [11]:

- \|\|$_{r}$, respectively $\|\cdot\|_{m, r}$, is the norm of a scalar- or vector- or tensor-valued function with components in $L^{r}(\Omega)$, respectively in $W^{m, r}(\Omega)$.

- $\|\cdot\|_{r ; \partial \Omega}$ or $\|\cdot\|_{m, r} ; \partial \Omega$, is the norm of a scalar- or vector- or tensor-valued function with components in $L^{r}(\partial \Omega)$ or in $W^{m, r}(\partial \Omega)$. Similarly, $\|\cdot\|_{r ; \Omega^{\prime}}$ or $\|\cdot\|_{m, r} ; \Omega^{\prime}$ denote the norms of functions in $L^{r}\left(\Omega^{\prime}\right)$ or in $W^{m, r}\left(\Omega^{\prime}\right)$ in the case when $\Omega^{\prime} \neq \Omega$.

- Vector functions or spaces of vector functions and their duals are denoted by boldface letters.

- $\boldsymbol{L}_{\sigma}^{2}(\Omega)$ is the closure of $\left\{\boldsymbol{u} \in \boldsymbol{C}_{0}^{\infty}(\Omega)\right.$; $\operatorname{div} \boldsymbol{u}=0$ in $\left.\Omega\right\}$ in $\boldsymbol{L}^{2}(\Omega)$. It coincides with the space $\left\{\boldsymbol{u} \in \boldsymbol{L}^{2}(\Omega)\right.$; $\operatorname{div} \boldsymbol{u}=0$ in $\Omega$ in the sense of distributions and $\left.\boldsymbol{u} \cdot \boldsymbol{n}\right|_{\partial \Omega}=0$ in the sense of traces $\}$. The orthogonal complement to $\boldsymbol{L}_{\sigma}^{2}(\Omega)$ in $\boldsymbol{L}^{2}(\Omega)$ consists of functions of the type $\nabla q$ for $q \in W^{1,2}(\Omega)$.

- $\boldsymbol{V}$ denotes the space of all divergence-free vector functions in $\boldsymbol{W}_{0}^{1,2}(\Omega)$. It is a subspace of $\boldsymbol{W}_{0}^{1,2}(\Omega)$.

- $\boldsymbol{D}^{1}=\left\{\boldsymbol{u} \in \boldsymbol{W}^{1,2}(\Omega) \cap \boldsymbol{L}_{\sigma}^{2}(\Omega) ;\left.\operatorname{curl} \boldsymbol{u} \cdot \boldsymbol{n}\right|_{\partial \Omega}=0\right.$ in the sense of traces $\}=$ $\left\{\boldsymbol{u}=\boldsymbol{u}_{0}+\nabla \varphi ; \boldsymbol{u}_{0} \in \boldsymbol{W}_{0}^{1,2}(\Omega), \Delta \varphi=-\nabla \cdot \boldsymbol{u}_{0}\right.$ in $\Omega$ and $\left.\partial \varphi /\left.\partial \boldsymbol{n}\right|_{\partial \Omega}=0\right\}=P_{\sigma} \boldsymbol{W}_{0}^{1,2}(\Omega)$ (where $P_{\sigma}$ is the orthogonal projection of $\boldsymbol{L}^{2}(\Omega)$ onto $\boldsymbol{L}_{\sigma}^{2}(\Omega)$ ).

- $\mathcal{R}=\left.\operatorname{curl}\right|_{D^{1}} ; \mathcal{R}$ is a self-adjoint operator in $\boldsymbol{L}_{\sigma}^{2}(\Omega)$.

- The equation $\mathcal{R} \boldsymbol{u}=\boldsymbol{f}$ (for $\boldsymbol{f} \in \boldsymbol{L}_{\sigma}^{2}(\Omega)$ ) has a unique solution $\boldsymbol{u} \in \boldsymbol{D}^{1}$ such that

$$
\|\boldsymbol{u}\|_{1,2} \leq c_{1}\|\boldsymbol{f}\|_{2}
$$

where constant $c_{1}$ is independent of $\boldsymbol{f}$. (See O. A. Ladyzhenskaya, V. A. Solonnikov [9].)

- There exist constants $c_{2}, c_{3}>0$ such that

$$
c_{2}\|\mathcal{R} \boldsymbol{u}\|_{2} \leq\|\boldsymbol{u}\|_{1,2} \leq c_{3}\|\mathcal{R} \boldsymbol{u}\|_{2} \text { for all } \boldsymbol{u} \in \boldsymbol{D}^{1} .
$$


- $\langle., .\rangle_{\partial \Omega}$ is the duality between $W^{-1 / 2,2}(\partial \Omega)$ and $W^{1 / 2,2}(\partial \Omega)$ (or between $\boldsymbol{W}^{-1 / 2,2}(\partial \Omega$ ) and $\left.\boldsymbol{W}^{1 / 2,2}(\partial \Omega)\right)$. Similarly, $\langle., .\rangle_{\Gamma_{i}}$ is the duality between $W^{-1 / 2,2}\left(\Gamma_{i}\right)$ and $W^{1 / 2,2}\left(\Gamma_{i}\right)$.

- By a neighborhood of $\partial \Omega$ we always mean an "interior neighborhood". More precisely, the $r$-neighborhood of $\partial \Omega$ is the open set $\Omega_{r}:=U_{r}(\partial \Omega) \cap \Omega$.

LEMmA 2.1. Given functions $\alpha_{0} \in W^{1 / 2,2}(\partial \Omega)$ and $\alpha_{1} \in W^{-1 / 2,2}(\partial \Omega)$ such that

$$
\int_{\partial \Omega} \alpha_{0} \mathrm{~d} S=0 \quad \text { and } \quad\left\langle\alpha_{1}, 1\right\rangle_{\Gamma_{i}}=0 \quad(i=0,1, \ldots, N),
$$

there exists a vector function $\boldsymbol{a} \in \boldsymbol{W}^{1,2}(\Omega)$ such that $\operatorname{div} \boldsymbol{a}=0$ a.e. in $\Omega, \boldsymbol{a}$ is harmonic (in the distributional sense) in some neighborhood of $\partial \Omega$ and

$$
\text { (a) }\left.\boldsymbol{a} \cdot \boldsymbol{n}\right|_{\partial \Omega}=\alpha_{0}, \quad \text { (b) }\left.\operatorname{curl} \boldsymbol{a} \cdot \boldsymbol{n}\right|_{\partial \Omega}=\alpha_{1} .
$$

Moreover, there exists a constant $c_{4}>0$, independent of $\alpha_{0}$ and $\alpha_{1}$, such that

$$
\|\boldsymbol{a}\|_{1,2} \leq c_{4}\left(\left\|\alpha_{0}\right\|_{1 / 2,2 ; \partial \Omega}+\left\|\alpha_{1}\right\|_{-1 / 2,2 ; \partial \Omega}\right)
$$

Proof. (i) First we solve the Neumann problem

$$
\Delta \psi_{1}=0 \quad \text { in } \Omega,\left.\quad \frac{\partial \psi_{1}}{\partial \boldsymbol{n}}\right|_{\partial \Omega}=\alpha_{1} .
$$

There exists a unique (up to an additive constant) weak solution $\psi_{1} \in W^{1,2}(\Omega)$ which depends continuously on $\alpha_{1}$ in the sense that

$$
\left\|\nabla \psi_{1}\right\|_{2} \leq c_{5}\left\|\alpha_{1}\right\|_{-1 / 2,2 ; \partial \Omega}
$$

where $c_{5}$ is independent of $\alpha_{1}$.

(ii) Next we consider the problem

$$
\operatorname{curl} \varphi_{0}=\nabla \psi_{1} \quad \text { in } \Omega,\left.\quad \varphi_{0}\right|_{\partial \Omega}=\mathbf{0} .
$$

Since $\left\langle\alpha_{1}, 1\right\rangle_{\Gamma_{i}}=0(i=0,1, \ldots, N)$, the flux of $\nabla \psi_{1}$ through each component of $\partial \Omega$ equals zero. Thus, due to [2], Theorem 2.1, the problem (18) is solvable in $\boldsymbol{W}_{0}^{1,2}(\Omega)$. Moreover, there exists $c_{6}>0$, independent of $\nabla \psi_{1}$, such that

$$
\left\|\boldsymbol{\varphi}_{0}\right\|_{1,2} \leq c_{6}\left\|\nabla \psi_{1}\right\|_{2} \text {. }
$$

(iii) Now we solve the Neumann problem

$$
\Delta \psi_{0}=-\operatorname{div} \varphi_{0} \quad \text { in } \Omega,\left.\quad \frac{\partial \psi_{0}}{\partial \boldsymbol{n}}\right|_{\partial \Omega}=\alpha_{0} .
$$

This problem has a unique (up to an additive constant) solution $\psi_{0} \in W^{2,2}(\Omega)$ which satisfies the estimate

$$
\left\|\nabla \psi_{0}\right\|_{1,2} \leq c_{7}\left(\left\|\varphi_{0}\right\|_{1,2}+\left\|\alpha_{0}\right\|_{1 / 2,2 ; \partial \Omega}\right)
$$

where $c_{5}$ is independent of $\varphi_{0}$ and $\alpha_{0}$, see e.g. [6], p. 15 .

Finally we put $\boldsymbol{a}:=\nabla \psi_{0}+\boldsymbol{\varphi}_{0}$. The inequalities (17), (19) and (21) imply (15). The function $\boldsymbol{a}$ is divergence-free because $\psi_{0}$ satisfies the equation (20). It is harmonic because $\operatorname{curl}^{2} \boldsymbol{a}=\operatorname{curl} \nabla \psi_{1}=\mathbf{0}$ in the sense of distributions in $\Omega$. The normal component of $\boldsymbol{a}$ on $\partial \Omega$ equals $\alpha_{0}$ because $\boldsymbol{a} \cdot \boldsymbol{n}=\nabla \psi_{0} \cdot \boldsymbol{n}=\alpha_{0}$ on $\partial \Omega$. Since $\operatorname{curl} \boldsymbol{a}=\operatorname{curl} \boldsymbol{\varphi}_{0}=\nabla \psi_{1}$ and consequently, $\operatorname{curl} \boldsymbol{a} \cdot \boldsymbol{n}=\nabla \psi_{1} \cdot \boldsymbol{n}=\alpha_{1}$ on $\partial \Omega$, the function $\boldsymbol{a}$ also satisfies (14b). 
Note that the function $\boldsymbol{a}$ with all the properties named in the lemma is not given uniquely. The construction described above leads to the function $\boldsymbol{a}$ which is harmonic not only in a neighborhood of $\partial \Omega$, but in the whole domain $\Omega$.

LEMma 2.2. Given $\beta \in W^{-1 / 2,2}(\partial \Omega)$ such that

$$
\langle\beta, 1\rangle_{\Gamma_{i}}=0 \quad(i=0,1, \ldots, N),
$$

there exists a function $\boldsymbol{v} \in \boldsymbol{D}^{1}$ such that

$$
\left.\frac{\partial \boldsymbol{v}}{\partial \boldsymbol{n}} \cdot \boldsymbol{n}\right|_{\partial \Omega}=\beta
$$

Proof. (i) We solve the equation

$$
\Delta_{\tau} \varphi=-\beta
$$

on each component $\partial \Omega$. Here $\Delta_{\tau}$ is the Laplace-Beltrami operator acting on functions defined on $\partial \Omega$. Using the representation of the surfaces $\Gamma_{i}(i=0,1, \ldots, N)$ by means of a finite number of local mappings of the class $C^{2,1}$ and the subordinate partition of unity, we can transform equation (23) to a finite number of elliptic equations with 2D generalized Laplace operators in $\mathbb{R}^{2}$. Solving them and summing the results, one can prove that the equation (23) has a solution $\varphi \in W^{3 / 2,2}(\partial \Omega)$. The solution is not unique, but it can be chosen so that

$$
\|\varphi\|_{3 / 2,2 ; \partial \Omega} \leq c_{8}\|\beta\|_{-1 / 2,2 ; \partial \Omega}
$$

where the constant $c_{8}$ is independent of $\beta$.

(ii) We solve the biharmonic equation $\Delta^{2} \zeta=0$ in $\Omega$ with the boundary conditions

$$
\text { (a) }\left.\zeta\right|_{\partial \Omega}=\varphi, \quad \text { (b) }\left.\frac{\partial \zeta}{\partial \boldsymbol{n}}\right|_{\partial \Omega}=0
$$

Due to [6], p. 16, this problem has a unique solution $\zeta \in W^{2,2}(\Omega)$ which depends continuously on $\varphi$ in the sense that

$$
\|\zeta\|_{2,2} \leq c_{9}\|\varphi\|_{3 / 2,2 ; \partial \Omega}
$$

where the constant $c_{9}$ does not depend on $\varphi$.

(iii) Let us observe that $-\Delta \zeta \in L_{0}^{2}(\Omega)$ where $L_{0}^{2}(\Omega)=\left\{p \in L^{2}(\Omega) ; \int_{\Omega} p \mathrm{~d} \boldsymbol{x}=0\right\}$. Then we can find $\boldsymbol{v}_{0} \in \boldsymbol{W}_{0}^{1,2}(\Omega)$ such that

$$
\operatorname{div} \boldsymbol{v}_{0}=-\Delta \zeta
$$

in $\Omega$. Indeed, by [6], Corollary 2.4, the operator div is an isomorphism of the space $\boldsymbol{V}^{\perp}$ onto $L_{0}^{2}(\Omega)$, where $\boldsymbol{V}^{\perp}$ is the orthogonal complement to $\boldsymbol{V}$ in $\boldsymbol{W}_{0}^{1,2}(\Omega)$. Thus, an appropriate $\boldsymbol{v}_{0}$ exists and its norm can be estimated:

$$
\left\|\boldsymbol{v}_{0}\right\|_{1,2} \leq c_{10}\|\Delta \zeta\|_{2}
$$

where $c_{10}$ is independent of $\zeta$.

(iv) Finally, we put $\boldsymbol{v}:=\boldsymbol{v}_{0}+\nabla \zeta$. The function $\boldsymbol{v}$ is divergence-free, its normal component on $\Omega$ is zero due to $(25 \mathrm{~b})$, its curl equals curl $\boldsymbol{v}_{0}$ and the normal component of this function equals zero because $\boldsymbol{v}_{0}=\mathbf{0}$ on $\partial \Omega$.

It remains to show that $\boldsymbol{v}$ satisfies (22). Due to the assumed smoothness of $\partial \Omega$, the function $\boldsymbol{n}$ can be extended from $\partial \Omega$ to a function of the class $C^{2}$ in the whole closure 
$\bar{\Omega}$. It can easily be observed that $\nabla \boldsymbol{v} \cdot \boldsymbol{n}$ is in $\boldsymbol{L}^{2}(\Omega)$ and its divergence is in $L^{2}(\Omega)$. Thus, $\boldsymbol{n} \cdot \nabla \boldsymbol{v} \cdot \boldsymbol{n}$ has a trace in $W^{-1 / 2,2}(\partial \Omega)$, which is exactly what we understand by $(\partial \boldsymbol{v} / \partial \boldsymbol{n}) \cdot \boldsymbol{n}$ on $\partial \Omega$. Clearly, since $\operatorname{div} \boldsymbol{v}=0$ in $\Omega$, the function $\operatorname{div} \boldsymbol{v}-(\partial \boldsymbol{v} / \partial \boldsymbol{n}) \cdot \boldsymbol{n}$ also has a trace in $W^{-1 / 2,2}(\partial \Omega)$.

We shall further denote by $\boldsymbol{n}$ not only the outer normal vector on $\partial \Omega$, but also its $C^{2}$ extension in $\bar{\Omega}$.

Suppose that $\left\{\varphi^{k}\right\}$ is a sequence in $W^{5 / 2,2}(\partial \Omega)$ which converges to $\varphi$ in $W^{3 / 2,2}(\partial \Omega)$ as $k \rightarrow+\infty$. Then the series of problems, given by the equations $\Delta^{2} \zeta^{k}=0$ in $\Omega$ and the boundary conditions

$$
\text { (a) }\left.\quad \zeta^{k}\right|_{\partial \Omega}=\varphi^{k}, \quad \text { (b) }\left.\frac{\partial \zeta^{k}}{\partial \boldsymbol{n}} \cdot \boldsymbol{n}\right|_{\partial \Omega}=0 .
$$

has solutions $\zeta^{k} \in W^{3,2}(\Omega)$, such that $\zeta^{k} \rightarrow \zeta$ in $W^{2,2}(\Omega)$ as $k \rightarrow+\infty$, see [6], p. 17. The series of problems $\operatorname{div} \boldsymbol{v}_{0}^{k}=-\Delta \zeta^{k}$ in $\Omega, \boldsymbol{v}_{0}^{k}=\mathbf{0}$ on $\partial \Omega$ has solutions $\boldsymbol{v}_{0}^{k} \in \boldsymbol{W}^{2,2}(\Omega)$ such that $\boldsymbol{v}_{0}^{k} \rightarrow \boldsymbol{v}_{0}$ in $\boldsymbol{W}_{0}^{1,2}(\Omega)$ as $k \rightarrow+\infty$, see [2], Theorem 2.1. Thus,

$$
\operatorname{div} \boldsymbol{v}^{k}-\frac{\partial \boldsymbol{v}^{k}}{\partial \boldsymbol{n}} \cdot \boldsymbol{n} \rightarrow \operatorname{div} \boldsymbol{v}-\frac{\partial \boldsymbol{v}}{\partial \boldsymbol{n}} \cdot \boldsymbol{n} \quad \text { in } W^{-1 / 2,2}(\Omega) \quad \text { as } k \rightarrow+\infty .
$$

The functions on the left hand side can be written as

$$
\operatorname{div} \boldsymbol{v}^{k}-\frac{\partial \boldsymbol{v}^{k}}{\partial \boldsymbol{n}} \cdot \boldsymbol{n}=\operatorname{div} \boldsymbol{v}_{0}^{k}-\frac{\partial \boldsymbol{v}_{0}^{k}}{\partial \boldsymbol{n}} \cdot \boldsymbol{n}+\Delta \zeta^{k}-\frac{\partial^{2} \zeta^{k}}{\partial \boldsymbol{n}^{2}}
$$

The first two terms on the right hand side have the trace on $\partial \Omega$ equal to zero because after the subtraction of $\left(\partial \boldsymbol{v}_{0}^{k} / \partial \boldsymbol{n}\right) \cdot \boldsymbol{n}$ from $\operatorname{div} \boldsymbol{v}_{0}^{k}$ on $\partial \Omega$, we have the sum of two tangential components of $\boldsymbol{v}^{k}$ differentiated in the tangential directions, which equals zero because $\boldsymbol{v}_{0}^{k}=\mathbf{0}$ on $\partial \Omega$. The trace of the second two terms on the right hand side can be simplified to $\Delta_{\tau} \zeta^{k}$, which equals $\Delta_{\tau} \varphi^{k}$ because $\varphi^{k}$ is the trace of $\zeta^{k}$ on $\partial \Omega$. Thus, the right hand side of (30) is equal to the limit of $\Delta_{\tau} \varphi^{k}$ as $k \rightarrow+\infty$ in the space $W^{-1 / 2,2}(\partial \Omega)$. However, due to the assumptions on the functions $\varphi^{k}$, the limit equals $\beta$.

LEMmA 2.3. Given $\alpha_{0} \in W^{1 / 2,2}(\partial \Omega), \alpha_{1} \in W^{-1 / 2,2}(\partial \Omega)$ and $\alpha_{2} \in W^{-1 / 2,2}(\partial \Omega)$ such that

$$
\int_{\partial \Omega} \alpha_{0} \mathrm{~d} S=0 \quad \text { and } \quad\left\langle\alpha_{1}, 1\right\rangle_{\Gamma_{i}}=\left\langle\alpha_{2}, 1\right\rangle_{\Gamma_{i}}=0 \quad(i=0,1, \ldots, N),
$$

there exists a vector function $\boldsymbol{a} \in \boldsymbol{W}^{1,2}(\Omega)$ such that $\operatorname{div} \boldsymbol{a}=0$ a.e. in $\Omega$ and

$$
\text { (a) }\left.\boldsymbol{a} \cdot \boldsymbol{n}\right|_{\partial \Omega}=\alpha_{0},
$$

(b) $\left.\operatorname{curl} \boldsymbol{a} \cdot \boldsymbol{n}\right|_{\partial \Omega}=\alpha_{1}$,

(c) $\left.\frac{\partial \boldsymbol{a}}{\partial \boldsymbol{n}} \cdot \boldsymbol{n}\right|_{\partial \Omega}=\alpha_{2}$.

Moreover, there exists $c_{11}>0$, independent of $\alpha_{0}, \alpha_{1}$ and $\alpha_{2}$, such that

$$
\|\boldsymbol{a}\|_{1,2} \leq c_{11}\left(\left\|\alpha_{0}\right\|_{1 / 2,2 ; \partial \Omega}+\left\|\alpha_{1}\right\|_{-1 / 2,2 ; \partial \Omega}+\left\|\alpha_{2}\right\|_{-1 / 2,2 ; \partial \Omega}\right) .
$$

Proof. Denote by $\boldsymbol{a}^{\prime}$ the function given by Lemma 2.1. Using the same arguments as in the final part of the proof of Lemma 2.2 , we can give the right sense to $\left(\partial \boldsymbol{a}^{\prime} / \partial \boldsymbol{n}\right) \cdot \boldsymbol{n}$ and we can show that it is an element of $W^{-1 / 2,2}(\partial \Omega)$. Since $\boldsymbol{a}^{\prime}$ belongs to the closure of $\left\{\boldsymbol{\phi} \in \boldsymbol{C}^{\infty}(\bar{\Omega}) ; \operatorname{div} \boldsymbol{\phi}=0\right\}$ in $\boldsymbol{W}^{1,2}(\Omega)$, it can be approximated by a sequence $\left\{\boldsymbol{a}^{k}\right\}$ in $\boldsymbol{C}^{\infty}(\bar{\Omega})$ such that $\operatorname{div} \boldsymbol{a}^{k}=0$, which converges to $\boldsymbol{a}^{\prime}$ in $\boldsymbol{W}^{1,2}(\Omega)$. Since $\boldsymbol{a}^{k}$ is divergencefree and due to the higher smoothness of $\left(\partial \boldsymbol{a}^{k} / \partial \boldsymbol{n}\right) \cdot \boldsymbol{n}$, the trace of the latter on $\partial \Omega$ 
equals $-\nabla_{\tau} \cdot \boldsymbol{a}_{\tau}^{k}$ where $\nabla_{\tau}$ is the "tangential" operator nabla (i.e. the $2 \mathrm{D}$ nabla operator acting in the tangent plane to $\partial \Omega$ ) and $\boldsymbol{a}_{\tau}^{k}$ is the tangential component of $\boldsymbol{a}^{k}$. Integrating on an arbitrary component $\Gamma_{i}$ of $\partial \Omega$, we can verify that the mean value of $\nabla_{\tau} \cdot \boldsymbol{a}_{\tau}^{k}$ on $\Gamma_{i}$ equals zero. Thus, we also have

$$
\left\langle\left(\partial \boldsymbol{a}^{\prime} / \partial \boldsymbol{n}\right) \cdot \boldsymbol{n}, 1\right\rangle_{\Gamma_{i}}=\lim _{k \rightarrow+\infty} \int_{\Gamma_{i}}\left(\partial \boldsymbol{a}^{k} / \partial \boldsymbol{n}\right) \cdot \boldsymbol{n} \mathrm{d} S=0 .
$$

Put $\beta:=\alpha_{2}-\left(\partial \boldsymbol{a}^{\prime} / \partial \boldsymbol{n}\right) \cdot \boldsymbol{n}$. Then $\beta \in W^{-1 / 2,2}(\partial \Omega)$ and $\langle\beta, 1\rangle_{\Gamma_{i}}=0(i=0,1, \ldots, N)$. Due to Lemma 2.2, there exists a function $\boldsymbol{v} \in \boldsymbol{D}^{1}$, satisfying (22). Then the function $\boldsymbol{a}:=\boldsymbol{a}^{\prime}+\boldsymbol{v}$ has all the properties named in the statement of the lemma. Note that, in contrast with function $\boldsymbol{a}^{\prime}$, the function $\boldsymbol{a}$ need not be harmonic in a neighborhood of $\partial \Omega$ because $\boldsymbol{v}$ is generally not harmonic in the neighborhood of $\partial \Omega$.

3. The weak problem with the inhomogeneous boundary conditions (9). Suppose that $\alpha_{0}, \alpha_{1}$ and $\alpha_{2}$ satisfy the assumptions of Lemma 2.1 and Lemma 2.3 and $\boldsymbol{a}$ is a function whose existence is given by Lemma 2.3. Solution of the equations (1), (2), satisfying the boundary conditions (9) can be sought for in the form $\boldsymbol{u}=\boldsymbol{a}+\boldsymbol{v}$ where $\boldsymbol{v}$ satisfies the equations

$$
\begin{aligned}
-\nu \Delta \boldsymbol{v}+(\boldsymbol{a} \cdot \nabla) \boldsymbol{v}+(\boldsymbol{v} \cdot \nabla) \boldsymbol{a}+(\boldsymbol{v} \cdot \nabla) \boldsymbol{v}+\nabla p & =\boldsymbol{g}, \\
\operatorname{div} \boldsymbol{v} & =0,
\end{aligned}
$$

in $\Omega$ and the homogeneous boundary conditions (5) on $\partial \Omega$. Here $\boldsymbol{g}=\boldsymbol{f}+\nu \Delta \boldsymbol{a}-(\boldsymbol{a} \cdot \nabla) \boldsymbol{a}$. However, we have already mentioned that (5) (for divergence-free vector functions) is equivalent to the homogeneous Dirichlet boundary condition

$$
\left.\boldsymbol{v}\right|_{\partial \Omega}=\mathbf{0} .
$$

Thus, the equations (33) and (34) can be solved with this usual boundary condition. If we find a solution $\boldsymbol{v}$ then the sum $\boldsymbol{u}=\boldsymbol{v}+\boldsymbol{a}$ is a solution of the problem (1), (2), (9).

The weak formulation of the problem (33)-(35) is well known, see e.g. O. A. Ladyzhenskaya [8], R. Temam [13], M. Feistauer [4] or G. P. Galdi [5].

Recall that the integral $\int_{\partial \Omega} \boldsymbol{a} \cdot \boldsymbol{n} \mathrm{d} S$, i.e. the flux of $\boldsymbol{a}$ through $\partial \Omega$, equals zero. While the authors assume that the fluxes of $\boldsymbol{a}$ through each component of $\partial \Omega$ separately equal zero in [8], [13] and [4], G. P. Galdi admits nonzero fluxes, which are however assumed to be "sufficiently small", in [5]. The reason is that the proof requires the quadratic form

$$
\mathcal{A}(\boldsymbol{v}, \boldsymbol{v}):=\int_{\Omega}[\nu \nabla \boldsymbol{v} \cdot \nabla \boldsymbol{v}+(\boldsymbol{v} \cdot \nabla) \boldsymbol{a} \cdot \boldsymbol{v}] \mathrm{d} \boldsymbol{x}
$$

to be coercive in space $\boldsymbol{V}$. It follows from [5], pp. 27-31, that if the flux of $\boldsymbol{a}$ through each component of $\partial \Omega$ is "small" then the function $\boldsymbol{a}$ can be modified in a sub-domain $\Omega^{\prime} \subset \overline{\Omega^{\prime}} \subset \Omega$ so that the condition of coerciveness of the form $\mathcal{A}$ is satisfied. If we denote the modified function again by $\boldsymbol{a}$, we thus obtain the lemma:

Lemma 3.1. There exists an $\epsilon>0$ such that IF $\alpha_{0} \in W^{1 / 2,2}(\partial \Omega)$ satisfies

$$
\sum_{i=1}^{N}\left|\int_{\Gamma_{i}} \alpha_{0} \mathrm{~d} S\right|<\epsilon
$$


$\alpha_{1}$ and $\alpha_{2}$ are elements of $W^{-1 / 2,2}(\partial \Omega)$ such that

$$
\left\langle\alpha_{1}, 1\right\rangle_{\Gamma_{i}}=\left\langle\alpha_{2}, 1\right\rangle_{\Gamma_{i}}=0 \quad(i=0,1, \ldots, N)
$$

Then there exists a vector function $\boldsymbol{a} \in \boldsymbol{W}^{1,2}(\Omega)$ such that $\operatorname{div} \boldsymbol{a}=0$ a.e. in $\Omega, \boldsymbol{a}$ satisfies the boundary conditions

$$
\text { (a) }\left.\boldsymbol{a} \cdot \boldsymbol{n}\right|_{\partial \Omega}=\alpha_{0}, \quad \text { (b) }\left.\operatorname{curl} \boldsymbol{a} \cdot \boldsymbol{n}\right|_{\partial \Omega}=\alpha_{1},
$$$$
\text { (c) }\left.\frac{\partial \boldsymbol{a}}{\partial \boldsymbol{n}} \cdot \boldsymbol{n}\right|_{\partial \Omega}=\alpha_{2}
$$

and the quadratic form (36) is coercive. Moreover, there exists $c_{12}>0$, independent of $\alpha_{0}, \alpha_{1}$ and $\alpha_{2}$, such that

$$
\|\boldsymbol{a}\|_{1,2} \leq c_{11}\left(\left\|\alpha_{0}\right\|_{1 / 2,2 ; \partial \Omega}+\left\|\alpha_{1}\right\|_{-1 / 2,2 ; \partial \Omega}+\left\|\alpha_{2}\right\|_{-1 / 2,2 ; \partial \Omega}\right) .
$$

With this function $\boldsymbol{a}$, following the procedure described in [5], pp. 16-18 and 33, we can prove that the boundary-value problem (33)-(35) has a weak solution $\boldsymbol{v}$. The solution depends continuously on the data in the sense that

$$
\|\boldsymbol{v}\|_{1,2} \leq c_{13}\left(\left\|\alpha_{0}\right\|_{1 / 2,2 ; \partial \Omega}+\left\|\alpha_{1}\right\|_{-1 / 2,2 ; \partial \Omega}+\left\|\alpha_{2}\right\|_{-1 / 2,2 ; \partial \Omega}+\|\boldsymbol{g}\|_{-1,2}\right) .
$$

where the constant $c_{13}$ is independent of $\alpha_{0}, \alpha_{1}, \alpha_{2}$ and $\boldsymbol{g}$. Here $\|\cdot\|_{-1,2}$ denotes the norm in the dual space to $\boldsymbol{V}$.

4. The weak problem with the inhomogeneous boundary conditions (10). In this section, it will be advantageous to write the equation (1) in the form

$$
\nu \operatorname{curl}^{2} \boldsymbol{u}+\operatorname{curl} \boldsymbol{u} \times \boldsymbol{u}+\nabla q=\boldsymbol{f}
$$

where $q=p+\frac{1}{2}|\boldsymbol{u}|^{2}$.

Suppose that $\alpha_{0}$ and $\alpha_{1}$ satisfy the assumptions of Lemma 2.1 and $\boldsymbol{a}$ is a function whose existence is given by this lemma. The weak solution $\boldsymbol{u}$ of the equations (41), (2), satisfying the boundary conditions (10), can be constructed in the form $\boldsymbol{u}=\boldsymbol{a}+\boldsymbol{v}$ where $\boldsymbol{v}$ satisfies in a weak sense the equations

$$
\begin{aligned}
\nu \operatorname{curl}^{2} \boldsymbol{v}+\operatorname{curl} \boldsymbol{a} \times \boldsymbol{v}+\operatorname{curl} \boldsymbol{v} \times \boldsymbol{a}+\operatorname{curl} \boldsymbol{v} \times \boldsymbol{v}+\nabla q & =\boldsymbol{g}, \\
\operatorname{div} \boldsymbol{v} & =0,
\end{aligned}
$$

(where $\boldsymbol{g}=\boldsymbol{f}-\nu \operatorname{curl}^{2} \boldsymbol{a}-\operatorname{curl} \boldsymbol{a} \times \boldsymbol{a}$ ) in $\Omega$ and the homogeneous boundary conditions $(8 \mathrm{a}),(8 \mathrm{~b})$ on $\partial \Omega$. This guarantees that $\boldsymbol{u}$ satisfies the conditions (10a) and (10b) on $\partial \Omega$, but it does not solve the question of validity of $(10 \mathrm{c})$. The reason why the condition (10c) cannot be treated in the same way as (10a) and (10b) is that (10c) involves the second derivatives of $\boldsymbol{u}$ and the construction of the weak solution $\boldsymbol{u}$ in $\boldsymbol{W}^{1,2}(\Omega)$ does not directly provide an opportunity to control $\operatorname{curl}^{2} \boldsymbol{u} \cdot \boldsymbol{n}$ on $\partial \Omega$. Thus, the boundary condition (10c) enters the weak formulation through a certain linear functional $\boldsymbol{b}$ which, in the case when the weak solution is "smooth", causes that it satisfies (10c) as a "natural boundary condition". This will be explained in greater detail later in this section. 
The weak formulation of the problem (42), (43), (8a), (8b) is: Suppose that $\boldsymbol{g} \in \boldsymbol{D}^{-1}$ (the dual to $\left.\boldsymbol{D}^{1}\right)$ and $\boldsymbol{b} \in \boldsymbol{W}^{-1 / 2,2}(\partial \Omega)$. We search for $\boldsymbol{v} \in \boldsymbol{D}^{1}$ such that

$$
\begin{gathered}
\int_{\Omega}[\nu \operatorname{curl} \boldsymbol{v} \cdot \operatorname{curl} \boldsymbol{\phi}+\operatorname{curl} \boldsymbol{a} \times \boldsymbol{v} \cdot \boldsymbol{\phi}+\operatorname{curl} \boldsymbol{v} \times \boldsymbol{a} \cdot \boldsymbol{\phi}+\operatorname{curl} \boldsymbol{v} \times \boldsymbol{v} \cdot \boldsymbol{\phi}] \mathrm{d} \boldsymbol{x} \\
=\langle\boldsymbol{g}, \boldsymbol{\phi}\rangle_{\Omega}+\langle\boldsymbol{b}, \boldsymbol{\phi}\rangle_{\partial \Omega}
\end{gathered}
$$

for all $\phi \in D^{1}$.

We shall further deal with the two questions:

1) In which sense does the weak formulation involve the boundary condition (10c)?

2) Existence of a weak solution and continuous dependence on the data.

1) Given $\boldsymbol{b} \in \boldsymbol{W}^{-1 / 2,2}(\partial \Omega)$, we define $\alpha_{2} \in W^{-3 / 2,2}(\partial \Omega)$ by the equation

$$
\nu\left\langle\alpha_{2}, \varphi\right\rangle_{\partial \Omega}^{*}=\langle\boldsymbol{b}, \nabla \varphi\rangle_{\partial \Omega}
$$

for $\varphi \in W^{2,2}(\Omega)$. Here $\langle., .\rangle_{\partial \Omega}^{*}$ denotes the duality between $W^{-3 / 2,2}(\partial \Omega)$ and $W^{3 / 2,2}(\partial \Omega)$.

If $\boldsymbol{g} \in \boldsymbol{L}_{\sigma}^{2}(\Omega)$ and $\boldsymbol{v}$ is a solution of (44) that belongs not only to $\boldsymbol{D}^{1}$, but also to $\boldsymbol{W}^{2,2}(\Omega)$, then we can first consider the test functions $\phi$ with a compact support in $\Omega$ and show that there exists a scalar function $q$ such that $\boldsymbol{v}, q$ satisfy the equations (42), (43) a.e. in $\Omega$. Then, following the standard procedure, we can consider all test functions from $\boldsymbol{D}^{1}$ and show, by means of the integration by parts in (44), that $\boldsymbol{v}$ satisfies

$$
\int_{\partial \Omega} \nu \operatorname{curl} \boldsymbol{v} \cdot(\boldsymbol{n} \times \boldsymbol{\phi}) \mathrm{d} S=\langle\boldsymbol{b}, \boldsymbol{\phi}\rangle_{\partial \Omega}
$$

for all $\phi \in D^{1}$. The function $\phi$, as an element of $\boldsymbol{D}^{1}$, can be written in the form

$$
\phi=\phi_{0}+\nabla \varphi
$$

where $\phi_{0} \in \boldsymbol{W}_{0}^{1,2}(\Omega)$ and $\varphi \in W^{2,2}(\Omega)$, see [1]. Recall that $\phi_{0}$ is a solution of the boundary-value problem (18) with the right hand side $\operatorname{curl} \phi$, i.e.

$$
\operatorname{curl} \phi_{0}=\operatorname{curl} \phi \quad \text { in } \Omega,\left.\quad \phi_{0}\right|_{\partial \Omega}=\mathbf{0} .
$$

The solution is not unique, however it can be chosen so that it satisfies

$$
\left\|\phi_{0}\right\|_{1,2} \leq c_{4}\|\operatorname{curl} \phi\|_{2} \leq c_{4} c_{1}\|\phi\|_{1,2}
$$

where $c_{4}$ is the constant from (19) and $c_{1}$ is the constant from (12). The function $\varphi$ is also not given uniquely by the decomposition (47), however $\nabla \varphi$ is defined uniquely on $\partial \Omega$. Substituting $\phi$ in the form (47) into the left hand side of (46), we obtain:

$$
\begin{aligned}
\int_{\partial \Omega} \operatorname{curl} \boldsymbol{v} \cdot(\boldsymbol{n} \times \boldsymbol{\phi}) \mathrm{d} S & =-\int_{\partial \Omega} \boldsymbol{n} \cdot(\operatorname{curl} \boldsymbol{v} \times \nabla \varphi) \mathrm{d} S=-\int_{\Omega} \operatorname{div}(\operatorname{curl} \boldsymbol{v} \times \nabla \varphi) \mathrm{d} \boldsymbol{x} \\
& =-\int_{\Omega} \operatorname{curl}^{2} \boldsymbol{v} \cdot \nabla \varphi \mathrm{d} \boldsymbol{x}=-\left\langle\operatorname{curl}^{2} \boldsymbol{v} \cdot \boldsymbol{n}, \varphi\right\rangle_{\partial \Omega}^{*} .
\end{aligned}
$$

Thus, (45) and (46) yield

$$
\nu\left\langle\alpha_{2}-\operatorname{curl}^{2} \boldsymbol{v} \cdot \boldsymbol{n}, \varphi\right\rangle_{\partial \Omega}^{*}=0 .
$$

This equation shows that $\boldsymbol{v}$ satisfies the boundary condition $\operatorname{curl}^{2} \boldsymbol{v} \cdot \boldsymbol{n}=\alpha_{2}$ in the sense of the equality in $W^{-3 / 2,2}(\partial \Omega)$. Since $\boldsymbol{u}=\boldsymbol{a}+\boldsymbol{v}$ and $\operatorname{curl}^{2} \boldsymbol{a}=0$ in the sense of distributions 
in some neighborhood of $\partial \Omega$, the function $\boldsymbol{u}$ also fulfills the boundary condition (10c) in the sense of equality in $W^{-3 / 2,2}(\partial \Omega)$.

2) It can be deduced from (13) that the operator $\mathcal{R}$, as an operator in $\boldsymbol{L}_{\sigma}^{2}(\Omega)$, has a compact resolvent. Consequently, its spectrum consists of a countable set of real eigenvalues. In order to prove the existence of a weak solution to the problem (42), (43), (8a), (8b), i.e. a solution $\boldsymbol{v}$ which satisfies (44), we can consider Galerkin approximations as linear combinations of the eigenfunctions of $\mathcal{R}$. Then we can again follow the well known procedure, see e.g. in [5] pp. 16-18, and show that the sequence of approximations contains a subsequence which converges to a weak solution of (42), (43), (8a), (8b). The only condition which we need is that the quadratic form

$$
\mathcal{A}(\boldsymbol{v}, \boldsymbol{v}):=\int_{\Omega}[\nu \operatorname{curl} \boldsymbol{v} \cdot \operatorname{curl} \boldsymbol{v}+\operatorname{curl} \boldsymbol{v} \times \boldsymbol{a} \cdot \boldsymbol{v}] \mathrm{d} \boldsymbol{x}
$$

is coercive in $\boldsymbol{D}^{1}$. The next crucial lemma says that if the prescribed fluxes through all components $\Gamma_{i}$ of $\partial \Omega$, which are given by the integrals of $\alpha_{0}$ on $\Gamma_{i}$, are sufficiently small then the function $\boldsymbol{a}$ can be chosen so that in addition to all the properties named in Lemma 2.1, the condition of coerciveness of the form $\mathcal{A}$ is fulfilled, too. The proof is rather technical and it is partially analogous to the procedure used in [5], pp. 28-31, however some steps require a different approach and a deeper analysis because we deal with functions $\boldsymbol{v}$ from $\boldsymbol{D}^{1}$ which need not be equal to zero on $\partial \Omega$, like in [5].

LEMMA 4.1. There exists $\epsilon>0$ such that IF $\alpha_{0}$ and $\alpha_{1}$ satisfy the assumptions of Lemma 2.1 and

$$
\sum_{i=1}^{N} \frac{1}{\operatorname{meas}_{2}^{1 / 2}\left(\Gamma_{i}\right)}\left|\int_{\Gamma_{i}} \alpha_{0} \mathrm{~d} S\right| \leq \epsilon
$$

Then there exists a function $\boldsymbol{a}$ with all the properties named in Lemma 2.1 and such that the quadratic form $\mathcal{A}$, defined by (51), is coercive in $\boldsymbol{D}^{1}$.

Proof. Recall that we consider $\boldsymbol{n}$ to be a $C^{2}$-extension of the outer normal vector from $\partial \Omega$ onto the whole $\bar{\Omega}$, see the proof of Lemma 2.2. Due to the smoothness of $\partial \Omega$, there exists $r>0$ such that the extension can be chosen so that it satisfies $\boldsymbol{n}(\boldsymbol{x})=\boldsymbol{n}(P \boldsymbol{x})$ for $\boldsymbol{x} \in \Omega_{r}=U_{r}(\partial \Omega) \cap \Omega$, where $P \boldsymbol{x}$ denotes the nearest point in $\partial \Omega$ to $\boldsymbol{x}$. (Px is uniquely defined for $r$ sufficiently small.) We further denote by $\boldsymbol{v}^{\tau}(\boldsymbol{x})$ the projection of $\boldsymbol{v}(\boldsymbol{x})$ onto the plane perpendicular to $\boldsymbol{n}(\boldsymbol{x})$ for $\boldsymbol{x} \in \Omega_{r}$ and $\boldsymbol{v} \in \boldsymbol{D}^{1}$. Thus, $\boldsymbol{v}^{\tau}(\boldsymbol{x})=$ $\boldsymbol{n}(\boldsymbol{x}) \times \boldsymbol{v}(\boldsymbol{x}) \times \boldsymbol{n}(\boldsymbol{x})$. We shall use a cut-off function $\eta_{\xi}(\boldsymbol{x}):=\eta(\delta(\boldsymbol{x}) / \xi)$ (for $\left.0<\xi<\frac{1}{2} r\right)$ where $\delta(\boldsymbol{x})$ denotes the distance of $\boldsymbol{x}$ from $\partial \Omega$ (hence $\delta(\boldsymbol{x})=|\boldsymbol{x}-P \boldsymbol{x}|$ for $\boldsymbol{x} \in \Omega_{r}$ ) and $\eta$ is an infinitely differentiable function with values in the interval $[0,1]$ such that

$$
\eta(t)=\left\{\begin{array}{lll}
1 & \text { for } & 0 \leq t \leq 1 \\
0 & \text { for } & 2 \leq t .
\end{array}\right.
$$

Let us denote $h_{i}:=\int_{\Gamma_{i}} \alpha_{0} \mathrm{~d} S$ and define $\chi_{i}(\boldsymbol{x}):=h_{i} / \operatorname{meas}_{2}\left(\Gamma_{i}\right)$ for $\boldsymbol{x} \in \Gamma_{i}$ and $i=0,1, \ldots, N$. Then $\chi_{i}$ is a constant function of $\Gamma_{i}$ and $\sum_{i=0}^{N} \int_{\Gamma_{i}} \chi_{i} \mathrm{~d} S=0$. Due to Lemma 2.1, there exists a function $\boldsymbol{h} \in \boldsymbol{W}^{1,2}(\Omega)$ such that $\operatorname{div} \boldsymbol{h}=0$ a.e. in $\Omega, \Delta \boldsymbol{h}=\mathbf{0}$ 
in the sense of distributions in some neighborhood of $\partial \Omega$ and

$$
\begin{gathered}
\text { (a) }\left.\boldsymbol{h} \cdot \boldsymbol{n}\right|_{\Gamma_{i}}=\chi_{i}, \quad \text { (b) }\left.\quad \operatorname{curl} \boldsymbol{h} \cdot \boldsymbol{n}\right|_{\Gamma_{i}}=0 \quad(i=0,1, \ldots, N), \\
\|\boldsymbol{h}\|_{1,2} \leq c_{4} \sum_{i=0}^{N}\left\|\chi_{i}\right\|_{1 / 2,2 ; \Gamma_{i}} \leq c_{4} \sum_{i=0}^{N}\left\|\chi_{i}\right\|_{2 ; \Gamma_{i}} \leq c_{4} \sum_{i=0}^{N} \frac{\left|h_{i}\right|}{\operatorname{meas}_{2}^{1 / 2}\left(\Gamma_{i}\right)} \leq c_{4} \epsilon .
\end{gathered}
$$

Let us define a function $\chi$ on $\partial \Omega$ by the equation $\chi(\boldsymbol{x}):=\chi_{i}(\boldsymbol{x})$ for $\boldsymbol{x} \in \Gamma_{i}(i=$ $0,1, \ldots, N)$. Applying once again Lemma 2.1 , we obtain a function $\boldsymbol{a}^{\prime}$ such that $\operatorname{div} \boldsymbol{a}^{\prime}=0$ a.e. in $\Omega, \Delta \boldsymbol{a}^{\prime}=\mathbf{0}$ in the sense of distributions in a neighborhood of $\partial \Omega$ and

$$
\begin{gathered}
\text { (a) } \begin{aligned}
\left.\boldsymbol{a}^{\prime} \cdot \boldsymbol{n}\right|_{\partial \Omega} & =\alpha_{0}-\chi, \quad \text { (b) }\left.\quad \operatorname{curl} \boldsymbol{a}^{\prime} \cdot \boldsymbol{n}\right|_{\partial \Omega}=\alpha_{1}, \\
\left\|\boldsymbol{a}^{\prime}\right\|_{1,2} & \leq c_{4}\left(\left\|\alpha_{0}-\chi\right\|_{1 / 2,2 ; \partial \Omega}+\left\|\alpha_{1}\right\|_{-1 / 2,2 ; \partial \Omega}\right) \\
& \leq c_{14}\left(\left\|\alpha_{0}\right\|_{1 / 2,2 ; \partial \Omega}+\left\|\alpha_{1}\right\|_{-1 / 2,2 ; \partial \Omega}\right) .
\end{aligned}
\end{gathered}
$$

The function $\boldsymbol{a}^{\prime}$ is divergence-free and its flux through each component $\Gamma_{i}$ of $\partial \Omega$ equals zero. Hence there exists a vector potential $\boldsymbol{\psi} \in \boldsymbol{W}^{2,2}(\Omega)$ such that $\boldsymbol{a}^{\prime}=\operatorname{curl} \boldsymbol{\psi}$ in $\Omega$. Moreover, there exists a constant $c_{15}>0$ independent of $\boldsymbol{a}^{\prime}$ and $\boldsymbol{\psi}$ such that

$$
\|\boldsymbol{\psi}\|_{2,2} \leq c_{15}\left\|\boldsymbol{a}^{\prime}\right\|_{1,2} \text {. }
$$

Finally, we put $\boldsymbol{a}:=\boldsymbol{h}+\boldsymbol{a}^{\prime \prime}$ where $\boldsymbol{a}^{\prime \prime}:=\operatorname{curl}\left(\eta_{\xi} \boldsymbol{\psi}\right)$. The exact value of $\xi$ will be specified later. Obviously, $\boldsymbol{a}$ coincides with $\boldsymbol{h}+\boldsymbol{a}^{\prime}$ in the neighborhood of $\partial \Omega$ and so $\boldsymbol{a}$ satisfies the boundary conditions (14) and it is harmonic (in the sense of distributions) in some neighborhood of $\partial \Omega$. From (54) and (56), one can deduce that $\boldsymbol{a}$ satisfies an estimate of the type (15).

Let us now deal with the question of coerciveness of the quadratic form $\mathcal{A}$. Due to (13), we have

$$
\mathcal{A}(\boldsymbol{v}, \boldsymbol{v})=\nu\|\mathcal{R} \boldsymbol{v}\|_{2}^{2}+\int_{\Omega} \boldsymbol{a} \times \boldsymbol{v} \cdot \operatorname{curl} \boldsymbol{v} \mathrm{d} \boldsymbol{x} \geq \frac{\nu}{c_{3}^{2}}\|\boldsymbol{v}\|_{1,2}^{2}-\left|\int_{\Omega} \operatorname{curl} \boldsymbol{v} \times \boldsymbol{a} \cdot \boldsymbol{v} \mathrm{d} \boldsymbol{x}\right| .
$$

By analogy with (47), the function $\boldsymbol{v}$ can be decomposed as the sum $\boldsymbol{v}_{0}+\nabla \varphi$, where $\boldsymbol{v}_{0} \in \boldsymbol{W}_{0}^{1,2}(\Omega)$ and $\varphi \in W^{2,2}(\Omega)$. Then

$$
\begin{aligned}
& \int_{\Omega} \operatorname{curl} \boldsymbol{v} \times \boldsymbol{a} \cdot \boldsymbol{v} \mathrm{d} \boldsymbol{x}=\int_{\Omega} \operatorname{curl} \boldsymbol{v}_{0} \times \boldsymbol{a} \cdot \boldsymbol{v} \mathrm{d} \boldsymbol{x}=\int_{\Omega} \operatorname{curl} \boldsymbol{v}_{0} \cdot \boldsymbol{a} \times \boldsymbol{v} \mathrm{d} \boldsymbol{x} \\
& =\int_{\Omega} \boldsymbol{v}_{0} \cdot \operatorname{curl}(\boldsymbol{a} \times \boldsymbol{v}) \mathrm{d} \boldsymbol{x}=\int_{\Omega} \boldsymbol{v}_{0} \cdot(\boldsymbol{v} \cdot \nabla) \boldsymbol{a} \mathrm{d} \boldsymbol{x}-\int_{\Omega} \boldsymbol{v}_{0} \cdot(\boldsymbol{a} \cdot \nabla) \boldsymbol{v} \mathrm{d} \boldsymbol{x} \\
& =\int_{\Omega} \boldsymbol{v}_{0} \cdot(\boldsymbol{v} \cdot \nabla) \boldsymbol{h} \mathrm{d} \boldsymbol{x}+\int_{\Omega} \boldsymbol{v}_{0} \cdot(\boldsymbol{v} \cdot \nabla) \boldsymbol{a}^{\prime \prime} \mathrm{d} \boldsymbol{x}-\int_{\Omega} \boldsymbol{v}_{0} \cdot(\boldsymbol{h} \cdot \nabla) \boldsymbol{v} \mathrm{d} \boldsymbol{x} \\
& \quad-\int_{\Omega} \boldsymbol{v}_{0} \cdot\left(\boldsymbol{a}^{\prime \prime} \cdot \nabla\right) \boldsymbol{v} \mathrm{d} \boldsymbol{x} .
\end{aligned}
$$

Denote by $I_{1}-I_{4}$ the integrals on the right hand side. In order to estimate $I_{1}$ and $I_{3}$, we shall use (54):

$$
\left|I_{1}+I_{3}\right| \leq C\left\|\boldsymbol{v}_{0}\right\|_{1,2}\|\boldsymbol{v}\|_{1,2}\|\boldsymbol{h}\|_{1,2} \leq C \epsilon\left\|\boldsymbol{v}_{0}\right\|_{1,2}\|\boldsymbol{v}\|_{1,2} \leq c_{16} \epsilon\|\boldsymbol{v}\|_{1,2}^{2} .
$$

The integral $I_{4}$ can be treated in the same way as the analogous term in [5], p. 31, and 
it can be shown that

$$
\begin{aligned}
\left|I_{4}\right| & \leq\|\boldsymbol{v}\|_{1,2}\left[\int_{\Omega}\left|\boldsymbol{v}_{0}\right|^{2}\left|\boldsymbol{a}^{\prime \prime}\right|^{2} \mathrm{~d} \boldsymbol{x}\right]^{\frac{1}{2}}=\|\boldsymbol{v}\|_{1,2}\left[\int_{\Omega}\left|\boldsymbol{v}_{0}\right|^{2}\left|\nabla \eta_{\xi} \times \boldsymbol{\psi}+\eta_{\xi} \operatorname{curl} \boldsymbol{\psi}\right|^{2} \mathrm{~d} \boldsymbol{x}\right]^{\frac{1}{2}} \\
& \leq\|\boldsymbol{v}\|_{1,2}\left[2 \int_{\Omega_{2 \xi}}\left|\boldsymbol{v}_{0}\right|^{2}\left|\eta^{\prime}\left(\frac{\delta(\boldsymbol{x})}{\xi}\right)\right|^{2} \frac{|\nabla \delta(\boldsymbol{x})|^{2}}{\xi^{2}}|\boldsymbol{\psi}|^{2} \mathrm{~d} \boldsymbol{x}+2 \int_{\Omega_{2 \xi}}\left|\boldsymbol{v}_{0}\right|^{2}|\operatorname{curl} \boldsymbol{\psi}|^{2} \mathrm{~d} \boldsymbol{x}\right]^{\frac{1}{2}} \\
& \leq C\|\boldsymbol{v}\|_{1,2}\left[\int_{\Omega_{2 \xi}} \frac{\left|\boldsymbol{v}_{0}\right|^{2}}{\delta(\boldsymbol{x})^{2}} \frac{\delta(\boldsymbol{x})^{2}}{\xi^{2}}|\boldsymbol{\psi}|^{2} \mathrm{~d} \boldsymbol{x}+\left\|\boldsymbol{v}_{0}\right\|_{1,2}^{2}\left(\int_{\Omega_{2 \xi}}\left|\boldsymbol{a}^{\prime}\right|^{3} \mathrm{~d} \boldsymbol{x}\right)^{\frac{2}{3}}\right]^{\frac{1}{2}} \\
& \leq C\|\boldsymbol{v}\|_{1,2}\|\boldsymbol{\psi}\|_{s, 2 ; \Omega_{2 \xi}}\left[\int_{\Omega} \frac{\left|\boldsymbol{v}_{0}\right|^{2}}{\delta(\boldsymbol{x})^{2}} \mathrm{~d} \boldsymbol{x}\right]^{\frac{1}{2}}+C\|\boldsymbol{v}\|_{1,2}\left\|\boldsymbol{v}_{0}\right\|_{1,2}\left\|\boldsymbol{a}^{\prime}\right\|_{3 ; \Omega_{2 \xi}} \\
& \leq C\|\boldsymbol{v}\|_{1,2}\left\|\boldsymbol{v}_{0}\right\|_{1,2}\left[\operatorname{meas}_{3}^{\frac{2-s}{2 s}}\left(\Omega_{2 \xi}\right)\|\boldsymbol{\psi}\|_{2,2}+\operatorname{meas}_{3}^{1 / 2}\left(\Omega_{2 \xi}\right)\left\|\boldsymbol{a}^{\prime}\right\|_{6}\right] \\
& \leq c_{17}(\xi)\|\boldsymbol{v}\|_{1,2}^{2}\left\|\boldsymbol{a}^{\prime}\right\|_{1,2}
\end{aligned}
$$

where $\frac{3}{2}<s<2$ and $c_{17}(\xi) \rightarrow 0$ as $\xi \rightarrow 0$. (We have used the notation $\Omega_{2 \xi}=U_{2 \xi}(\partial \Omega) \cap \Omega$ and the estimates $\left\|\boldsymbol{v}_{0} / \delta(\boldsymbol{x})\right\|_{2} \leq C\left\|\boldsymbol{v}_{0}\right\|_{1,2}$, see [5], p. 31, and $\left\|\boldsymbol{v}_{0}\right\|_{1,2} \leq c_{4} c_{1}\|\boldsymbol{v}\|_{1,2}$, see (49).) Since $\boldsymbol{a}^{\prime \prime}=\mathbf{0}$ in $\Omega_{2 \xi}$, the integral $I_{2}$ can be estimated as follows:

$$
\begin{aligned}
\left|I_{2}\right| \leq & \left|\int_{\Omega_{2 \xi}}\left(\boldsymbol{v}-\boldsymbol{v}^{\tau}\right) \cdot \nabla \boldsymbol{a}^{\prime \prime} \cdot \boldsymbol{v}_{0} \mathrm{~d} \boldsymbol{x}\right|+\left|\int_{\Omega_{2 \xi}} \boldsymbol{v}^{\tau} \cdot \nabla \boldsymbol{a}^{\prime \prime} \cdot \boldsymbol{v}_{0} \mathrm{~d} \boldsymbol{x}\right| \\
\leq & \left|\int_{\Omega_{2 \xi}} \operatorname{div}\left(\boldsymbol{v}-\boldsymbol{v}^{\tau}\right) \boldsymbol{a}^{\prime \prime} \cdot \boldsymbol{v}_{0} \mathrm{~d} \boldsymbol{x}\right|+\left|\int_{\Omega_{2 \xi}}\left(\boldsymbol{v}-\boldsymbol{v}^{\tau}\right) \cdot \nabla \boldsymbol{v}_{0} \cdot \boldsymbol{a}^{\prime \prime} \mathrm{d} \boldsymbol{x}\right| \\
& +\left|\int_{\Omega_{2 \xi}} \boldsymbol{v}^{\tau} \cdot \nabla\left(\nabla \eta_{\xi} \times \boldsymbol{\psi}+\eta_{\xi} \operatorname{curl} \boldsymbol{\psi}\right) \cdot \boldsymbol{v}_{0} \mathrm{~d} \boldsymbol{x}\right| \leq I_{21}+I_{22}+I_{23}
\end{aligned}
$$

where

$$
\begin{aligned}
& I_{21}=\left\|\operatorname{div} \boldsymbol{v}^{\tau}\right\|_{2}\left(\int_{\Omega_{2 \xi}}\left|\boldsymbol{a}^{\prime \prime}\right|^{2}\left|\boldsymbol{v}_{0}\right|^{2} \mathrm{~d} \boldsymbol{x}\right)^{1 / 2}, \\
& I_{22}=\left\|\boldsymbol{v}_{0}\right\|_{1,2}\left(\int_{\Omega_{2 \xi}}\left|\boldsymbol{v}-\boldsymbol{v}^{\tau}\right|^{2}\left|\boldsymbol{a}^{\prime \prime}\right|^{2} \mathrm{~d} \boldsymbol{x}\right)^{1 / 2}, \\
& I_{23}=\left|\int_{\Omega_{2 \xi}} v_{j}^{\tau} \partial_{j}\left(\nabla \eta_{\xi} \times \boldsymbol{\psi}+\eta_{\xi} \operatorname{curl} \boldsymbol{\psi}\right) \cdot \boldsymbol{v}_{0} \mathrm{~d} \boldsymbol{x}\right| .
\end{aligned}
$$

The integrals in $I_{21}$ and $I_{22}$ can be treated in the same way as $I_{4}$. It is important that the functions $\boldsymbol{v}_{0}$ and $\boldsymbol{v}-\boldsymbol{v}^{\tau}$ have the trace on $\partial \Omega$ equal to zero and we can use the inequalities $\left\|\boldsymbol{v}_{0} / \delta(\boldsymbol{x})\right\|_{2} \leq C\left\|\boldsymbol{v}_{0}\right\|_{1,2}$ and $\left\|\left(\boldsymbol{v}-\boldsymbol{v}^{\tau}\right) / \delta(\boldsymbol{x})\right\|_{2} \leq C\left\|\boldsymbol{v}-\boldsymbol{v}^{\tau}\right\|_{1,2}$. Thus, we obtain

$$
\begin{aligned}
& I_{21} \leq c_{18}(\xi)\left\|\boldsymbol{v}^{\tau}\right\|_{1,2}\left\|\boldsymbol{v}_{0}\right\|_{1,2}\left\|\boldsymbol{a}^{\prime}\right\|_{1,2} \leq c_{18}(\xi) c_{19}\|\boldsymbol{v}\|_{1,2}^{2}\left\|\boldsymbol{a}^{\prime}\right\|_{1,2}, \\
& I_{22} \leq c_{18}(\xi)\left\|\boldsymbol{v}_{0}\right\|_{1,2}\left\|\boldsymbol{v}-\boldsymbol{v}^{\tau}\right\|_{1,2}\left\|\boldsymbol{a}^{\prime}\right\|_{1,2} \leq c_{18}(\xi) c_{20}\|\boldsymbol{v}\|_{1,2}^{2}\left\|\boldsymbol{a}^{\prime}\right\|_{1,2}
\end{aligned}
$$

where $c_{16}(\xi) \rightarrow 0$ for $\xi \rightarrow 0$. Finally, in order to estimate $I_{23}$, we use the identities $v_{j}^{\tau} \partial_{j} \nabla \eta_{\xi}=\nabla\left(v_{j}^{\tau} \partial_{j} \eta_{\xi}\right)-\nabla v_{j}^{\tau} \partial_{j} \eta_{\xi}=-\nabla v_{j}^{\tau} \partial_{j} \eta_{\xi}$ which hold because $v_{j}^{\tau} \partial_{j} \eta_{\xi}=\boldsymbol{v}^{\tau} \cdot \nabla \eta_{\xi}=$ 0 in $\Omega_{2 \xi}$. Furthermore, we also use the estimate $\|\boldsymbol{\psi}\|_{\infty ; \Omega_{2 \xi}} \leq C\|\boldsymbol{\psi}\|_{s, 2 ; \Omega_{2 \xi}}$ for some fixed 
$s$ such that $\frac{3}{2}<s<2$. Hence we get

$$
\begin{aligned}
I_{23}= & \mid \int_{\Omega_{2 \xi}}\left(-\partial_{j} \eta_{\xi} \nabla v_{j}^{\tau} \times \boldsymbol{\psi}+v_{j}^{\tau} \nabla \eta_{\xi} \times \partial_{j} \boldsymbol{\psi}+v_{j}^{\tau} \partial_{j} \eta_{\xi} \operatorname{curl} \boldsymbol{\psi}\right. \\
& \left.+v_{j}^{\tau} \eta_{\xi} \operatorname{curl}\left(\partial_{j} \boldsymbol{\psi}\right)\right) \cdot \boldsymbol{v}_{0} \mathrm{~d} \boldsymbol{x} \mid \\
\leq & C \int_{\Omega_{2 \xi}}\left|\nabla \eta_{\xi}\right|\left(\left|\nabla \boldsymbol{v}^{\tau}\right||\boldsymbol{\psi}|+\left|\boldsymbol{v}^{\tau}\right||\nabla \boldsymbol{\psi}|\right)\left|\boldsymbol{v}_{0}\right| \mathrm{d} \boldsymbol{x}+\int_{\Omega_{2 \xi}}\left|\boldsymbol{v}^{\tau}\right|\left|\nabla^{2} \boldsymbol{\psi}\right|\left|\boldsymbol{v}_{0}\right| \mathrm{d} \boldsymbol{x} \\
\leq & C \int_{\Omega_{2 \xi}} \frac{\left|\boldsymbol{v}_{0}\right|}{\delta(\boldsymbol{x})}\left(\left|\nabla \boldsymbol{v}^{\tau}\right||\boldsymbol{\psi}|+\left|\boldsymbol{v}^{\tau}\right||\nabla \boldsymbol{\psi}|\right) \mathrm{d} \boldsymbol{x}+C\left\|\boldsymbol{v}^{\tau}\right\|_{6}\|\boldsymbol{\psi}\|_{2,2}\left\|\boldsymbol{v}_{0}\right\|_{6} \operatorname{meas}_{3}^{1 / 6}\left(\Omega_{2 \xi}\right) \\
\leq & C\left\|\boldsymbol{v}_{0} / \delta(\boldsymbol{x})\right\|_{2}\left(\left\|\boldsymbol{v}^{\tau}\right\|_{1,2}\|\boldsymbol{\psi}\|_{s, 2 ; \Omega_{2 \xi}}+\left\|\boldsymbol{v}^{\tau}\right\|_{6}\|\nabla \boldsymbol{\psi}\|_{6} \operatorname{meas}_{3}^{1 / 6}\left(\Omega_{2 \xi}\right)\right) \\
& +C\left\|\boldsymbol{v}^{\tau}\right\|_{1,2}\|\boldsymbol{\psi}\|_{2,2}\left\|\boldsymbol{v}_{0}\right\|_{1,2} \operatorname{meas}_{3}^{1 / 6}\left(\Omega_{2 \xi}\right) \\
\leq & C\left\|\boldsymbol{v}_{0}\right\|_{1,2}\left\|\boldsymbol{v}^{\tau}\right\|_{1,2}\left(\|\boldsymbol{\psi}\|_{2,2 ; \Omega_{2 \xi}} \operatorname{meas}_{3}^{\frac{2-s}{2 s}}\left(\Omega_{2 \xi}\right)+\|\boldsymbol{\psi}\|_{2,2} \operatorname{meas}_{3}^{1 / 6}\left(\Omega_{2 \xi}\right)\right) \\
\leq & c_{21}(\xi)\|\boldsymbol{v}\|_{1,2}^{2}\left\|\boldsymbol{a}^{\prime}\right\|_{1,2}
\end{aligned}
$$

where $c_{21}(\xi) \rightarrow 0$ for $\xi \rightarrow 0$. Substituting all these inequalities to (58), denoting $c_{22}(\xi):=$ $c_{17}(\xi)+c_{18}(\xi)\left(c_{19}+c_{20}\right)+c_{21}(\xi)$ and estimating the norm $\left\|\boldsymbol{a}^{\prime}\right\|_{1,2}$ by means of $(56)$, we obtain

$$
\begin{aligned}
\mathcal{A}(\boldsymbol{v}, \boldsymbol{v}) & \geq\|\boldsymbol{v}\|_{1,2}^{2}\left[\frac{\nu}{c_{3}^{2}}-c_{16} \epsilon-\left(c_{17}(\xi)+c_{18}(\xi)\left(c_{19}+c_{20}\right)+c_{21}(\xi)\right)\left\|\boldsymbol{a}^{\prime}\right\|_{1,2}\right] \\
& \geq\|\boldsymbol{v}\|_{1,2}^{2}\left[\frac{\nu}{c_{3}^{2}}-c_{16} \epsilon-c_{22}(\xi) c_{14}\left(\left\|\alpha_{0}\right\|_{1 / 2,2 ; \partial \Omega}+\left\|\alpha_{1}\right\|_{-1 / 2,2 ; \partial \Omega}\right)\right] .
\end{aligned}
$$

We observe that the form $\mathcal{A}$ is coercive, indeed, if $\epsilon$ and $\xi$ are sufficiently small.

We have already mentioned that the coerciveness of the form $a$ enables us to prove the existence and appropriate estimates of the Galerkin approximations of a solution of $\boldsymbol{v}$ of the problem (44). The standard procedure, see e.g. [4], [5] or [13], finally leads to the theorem:

Theorem 4.1. Suppose that $\boldsymbol{g} \in \boldsymbol{D}^{-1}, \boldsymbol{b} \in \boldsymbol{W}^{-1 / 2,2}(\partial \Omega), \alpha_{0} \in W^{1 / 2,2}(\partial \Omega), \alpha_{1} \in$ $W^{-1 / 2,2}(\partial \Omega)$ and $\alpha_{0}$ satisfies the condition (52) of sufficiently small prescribed fluxes through the components of $\partial \Omega$. Let $\boldsymbol{a}$ be the function given by Lemma 2.1, constructed in accordance with Lemma 4.1 so that the quadratic form a, defined by (51), is coercive in $\boldsymbol{D}^{1}$. Then the weak problem (44) has a solution $\boldsymbol{v} \in \boldsymbol{D}^{1}$. Moreover, there exists a constant $c_{23}=c_{23}(\epsilon, \nu, \Omega)>0$ such that

$$
\|\boldsymbol{v}\|_{1,2} \leq c_{23}\left(\|\boldsymbol{g}\|_{-1,2}+\|\boldsymbol{b}\|_{-1 / 2,2 ; \partial \Omega}+\left\|\alpha_{0}\right\|_{1 / 2,2 ; \partial \Omega}+\left\|\alpha_{1}\right\|_{-1 / 2,2 ; \partial \Omega}\right)
$$

where $\|\cdot\|_{-1,2}$ denotes the norm in $\boldsymbol{D}^{-1}$.

Acknowledgements. The research was partially supported by the Grant Agency of the Czech Republic (grant No. 201/08/0012), by the research plan of the Ministry of Education of the Czech Republic No. MSM 6840770010, by the Nečas Center for Mathematical Modelling (project No. LC06052 financed by the Ministry of Education of the Czech Republic) and by the University of Sud-Toulon-Var. 


\section{References}

[1] H. Bellout, J. Neustupa and P. Penel, On the Navier-Stokes equation with boundary conditions based on vorticity, Math. Nachr. 269-270 (2004), 59-72.

[2] W. Borchers and H. Sohr, On the equations $\operatorname{rot} \boldsymbol{v}=\boldsymbol{g}$ and $\operatorname{div} \boldsymbol{v}=f$ with zero boundary conditions, Hokkaido Math. J. 19 (1990), 67-87.

[3] M. Bulíček, J. Málek and R. Rajagopal, Navier's slip and evolutionary Navier-Stokes like systems with pressure and shear-rate dependent viscosity, Indiana Univ. Math. J. 56 (2007), 51-85.

[4] M. Feistauer, Mathematical Methods in Fluid Dynamics, Pitman Monographs and Surveys in Pure and Applied Mathematics 67, Longman Scientific \& Technical, Harlow, 1993.

[5] G. P. Galdi, An Introduction to the Mathematical Theory of the Navier-Stokes Equations, Vol. II: Nonlinear Steady Problems, Springer Tracts in Natural Philosophy 39, Springer, New Yor, 1998.

[6] V. Girault and P.-A. Raviart, Finite Element Methods for Navier-Stokes Equations, Springer, Berlin, 1986.

[7] W. Jäger and A. Mikelič, On the roughness-induced effective boundary conditions for an incompressible viscous flow, J. Diff. Equations 170 (2001), 96-122.

[8] O. A. Ladyzhenskaya, The Mathematical Theory of Viscous Incompressible Flow, Gordon and Breach, New York, 1969.

[9] O. A. Ladyzhenskaya and V. A. Solonnikov, Solution of some non-stationary problems of magneto-hydrodynamics, Trudy Mat. Inst. V. A. Steklova 59 (1960), 115-173.

[10] J. Neustupa and P. Penel, Incompressible viscous fluid flows and the generalized impermeability boundary conditions, IASME Transactions 2 (2005), 1254-1261.

[11] J. Neustupa and P. Penel, On regularity of a weak solution to the Navier-Stokes equation with generalized impermeability boundary conditions, Nonlinear Analysis 66 (2007), 17531769.

[12] J. Neustupa, P. Penel and R. Picard, The Navier-Stokes equation with the generalized impermeability boundary conditions in a general domain, in preparation.

[13] R. Temam, Navier-Stokes Equations, North-Holland, Amsterdam, 1977.

[14] C. Truesdell, The mechanical foundations of elasticity and fluid dynamics, J. Rat. Mech. Anal. 1 (1952), 125-300.

[15] W. Zajączkowski, Global special regular solutions to the Navier-Stokes equations in a cylindrical domain under boundary slip conditions, Dissertationes Math. (Rozprawy Mat.) $432(2005)$. 
